REVISTA ECONOMÍA

Vol. 70, N. ${ }^{\circ}$ III (mayo 20I8), I73-I86

\title{
EL PENSAMIENTO MARXISTA Y SUS IMPLICACIONES PARA LA ECOLOGÍA APUNTES GENERALES
}

\author{
ÉDGAR ISCH L. \\ Universidad Central del Ecuador \\ Recepción de manuscrito: 20 de febrero de 2018 \\ Aceptación versión final: 13 abril de 2018
}

\begin{abstract}
RESUMEN El capitalismo es un sistema y una civilización en crisis que se caracteriza, entre otros aspectos fundamentales, por la explotación del trabajador, la superexplotación de la naturaleza y la enajenación del trabajo y los seres humanos que se miran al margen o por encima del mundo natural. Por ello, la crisis ambiental que vivimos hoy es parte de la crisis general del sistema. El marxismo entrega un método para entender esa realidad, que debe ser aplicado para analizar y enfrentar los problemas ecológicos, comprendiendo que la transformación social debe restituir el metabolismo entre sociedad y naturaleza. Elementos de ese método se consideran aquí.
\end{abstract}

pALABRAS ClAVE Marxismo, ecología, recursos naturales, ecología política.

ABSTRACT Capitalism is a system and a civilization in crisis characterized, among other fundamental aspects, by the exploitation of the worker, the super-exploitation of nature and the alienation of work and human beings looked at the margin or above the natural world. Therefore, the environmental crisis we are experiencing today is part of the general crisis of the system. Marxism provides a method to understand this reality, which must be applied to analyze and deal with ecological problems, understanding that social transformation must restore the metabolism between society and nature. Elements of that method considered here.

KEYWORDS Marxism, ecology, natural resources, political ecology.

Jel CODES B14, Q5, P28.

\section{INTRODUCCIÓN}

Ciertas lecturas del marxismo y del trabajo de Carlos Marx y Federico Engels muchas veces han adolecido de defectos de fondo y forma, especialmente cuando se han basado en opiniones de terceros sin llegar a revisar de manera directa sus escritos y su vida. En otros casos, se trató de acercamientos impulsados por un momento histórico preciso que marcaba la ruta de búsqueda y las prioridades de los investigadores, lo que ocultaba planteamientos realmente importantes, como cuando se centró las críticas en aspectos parciales - Levi, Wittwenstwein, Weber, Hayek, entre otros-. 
La crisis ambiental verificable en el cambio climático, la acelerada desaparición de especies y reducción de la biodiversidad, la reducción de fuentes de agua dulce segura, y el aumento de factores de riesgo para la humanidad, no es sino otra expresión de la crisis general del sistema capitalista. Como realidad, esta crisis obligó a realizar referencias a la obra marxiana, pero no siempre para comprenderla. El absurdo llega a que en charlas formales o conversaciones, como en una brindada por el autor de estos apuntes en la Universidad del Valle, Colombia, en 2013, se pretendiese negar el aporte de Marx a los estudios ecologistas preguntando «¿Qué dijo sobre el cambio climático?» Por supuesto que nada, pues la ciencia más avanzada de su época, en la que basó sus análisis, no podía aún predecir un fenómeno que, a pesar de antecedentes previos en 1896 Svante Arrhenius ya establece una relación entre el co2 y la temperatura del planeta-, no sería tema de mayor importancia en las publicaciones científicas hasta 1975, cuando Wallace Smith Broecker (1975) publicó el artículo científico: Cambio climático: ¿estamos al borde de un calentamiento global pronunciado? En el mismo grado de absurdo está la expresión de un ferviente extractivista, el expresidente Rafael Correa: «¿Dónde está en el Manifiesto Comunista el no a la minería? ¿Qué teoría socialista dijo no a la minería?» (entrevista a Rafael Correa, mayo 2012), a la que responderían intelectuales como Eduardo Gudinas (2013) que denunciara la absurda búsqueda de un Marx extractivista y Joan Martínez Alier (2013) quien criticara que no se entienda que Marx y Engels denunciaron al capitalismo depredador en su conjunto. El extractivismo es un problema central en el debate sobre el «desarrollo» y la ecología en América Latina, que no puede ser defendido con falacias como la expresada por Rafael Correa.

Pero hay otro factor a considerar: Marx y Engels trataron los más diversos temas de las ciencias, no solo de las sociales, pero el tiempo de una vida humana, por genial que sea, no permite profundizar en todas. Debieron principalizar ciertos temas como la crítica a la Economía Política, pero dejaron profundas huellas en la aplicación del materialismo histórico en otras áreas. Esto implica que más que repetir palabras de Marx y Engels sobre temas ecológicos, lo realmente importante es tratar de comprender su método y su aplicación para comprender esta temática. Método que, como dijera Engels (1886): «no es un dogma, sino una guía para la acción»; confirmándolo más adelante (1895): «... toda la concepción de Marx no es una doctrina, sino un método. No ofrece dogmas hechos, sino puntos de partida para la ulterior investigación y el método para dicha investigación».

Este artículo no pretende sino acercarse a la relación entre la ecología y el método marxista, considerando elementos fundamentales del mismo. Establecer la existencia de esa relación se conecta con identificar algunas líneas de pensamiento marxista que contribuyen a los debates actuales, a la comprensión de las contradicciones sociales en torno a la apropiación y distribución de los beneficios de la naturaleza y al desarrollo de las llamadas ciencias naturales en conexión con la realidad social de nuestros días.

\section{LA ECONOMÍA POLÍTICA Y SU RELACIÓN CON LA ECOLOGÍA}

El desarrollo de la ecología tiene una historia que empieza en el esfuerzo humano por conocer su entorno. Así nacieron ciencias como la geografía, la biología, la química y la economía que, en su expresión contemporánea, aportan a la Ecología Política. Es frecuente señalar 
la relación etimológica entre las palabras economía y ecología (Milián, 2007), cuyos orígenes vienen de la palabra griega oikos que se puede traducir como hogar o casa. Mientras la ecología procura el conocimiento de la casa, la economía se centraría en la administración de la misma. Por supuesto, cuando la economía se reduce a un ejercicio de contabilidad, pierde la amplitud social que le corresponde.

Como sucede con toda ciencia, la ecología presenta una historia de maduración y concreción. Ello ha conllevado que no se pueda considerar que exista una sola rama de la ecología. Así, desde cuando el zoólogo Ernst Haeckel (1866), usara por primera vez el término «ecología», en su libro Morfología general de los organismos, rápidamente se tendrían cuestionamientos sobre los límites biologicistas de su estudio centrado en las condiciones de vida de los seres vivos, y sobre cuál era el nivel de análisis correcto: especie, población, comunidad, ecosistema, biósfera y la interrelación entre ellos y el ambiente que habitan.

Particularmente en el siglo xx se dio lugar a otras corrientes de estudio de la ecología, que irían madurando y retroalimentándose (Isch, 2017), resultado además de la interdisciplina con las ciencias humanas. Así surgirán la Ecología Humana, Ecología Social, Ecología Urbana y la Ecología Política, motivo de este texto. De la última, hay diversas definiciones que encuentran puntos comunes como los planteados por Héctor Alimonda, uno de los más prestigiosos investigadores latinoamericano en este campo:

Parafraseando a Gramsci, puede decirse que para Lipietz todas las cuestiones ambientales significativas son políticas. Esto es así precisamente porque la particularidad de la ecología de la especie humana es que sus relaciones con la naturaleza están mediatizadas por formas de organización social, que reposan en dispositivos políticos para asegurar su consenso y su reproducción. (Alimonda, 2012, p. 8)

Según Anthony Bebbington (2007), los estudiosos de la ecología política presentan tres puntos de convergencia con las que mantiene diálogo continuo:

- La de las resistencias y movimientos sociales, que son respuestas populares a los procesos de despojo de las fuentes de supervivencia, y resalta el papel de los grupos humanos en la transformación de las condiciones estructurales. Para Martínez Alier (2005), esto haría de la ecología política una corriente de activismo ambiental, a su vez integrada por «el movimiento por la justicia ambiental, el ecologismo popular, el ecologismo de los pobres, nacidos de los conflictos ambientales a nivel local, regional, nacional y global causados por el crecimiento económico y la desigualdad social».

- La de la geografía humana y la antropología cultural, que ayudan a comprender los espacios como territorios socialmente construidos sobre una base material. Desde la antropología se resalta la relación entre cultura y naturaleza desde las diferentes sociedades y la manera en que dicha relación expresa un momento histórico y un territorio.

- La economía política, principalmente la marxista.

O dicho de manera simple, no hay forma de entender la ecología sin entender primero la economía política; es decir, especificando las relaciones de poder y de desigualdad que determinan quiénes tienen acceso a los recursos naturales, quiénes no y quiénes pueden definir el uso de estos recursos. (Bebbington, 2007, p. 28) 
Incluso para los esfuerzos de estudiar la ecología desde las ciencias naturales, esto resulta hoy importante, ya que podemos decir que no hay territorio o ecosistema que no esté afectado por las acciones humanas. El caso extremo que así lo demuestra es el cambio climático que ha alterado procesos naturales a nivel mundial y que se trata de consecuencia de actividades humanas relacionadas con un modelo de producción (IPCC, 2007; Isch, 2013).

\section{EL MATERIALISMO DIALÉCTICO Y LA NATURALEZA}

Marx y Engels, en primer lugar, plantean una visión no ontológica del materialismo, teniendo como punto de partida la materia (Castellanos Suárez, 2015). Si bien hay posturas que pretenden diferenciar a Marx y Engels al grado de contraponerlos en este punto llegando a identificar a Engels como metafísico y dogmático (Schmidt, 1976), así como en su filosofía general (Kolakowski, 1983) que llega al punto de plantear la distancia entre un «naturalismo engeliano» y un «humanismo marxista», se trata de perspectivas cuestionables (Piedra Arancibia, 2015) o propio de cuando «más que conocer a Marx se perseguía poseer a Marx» (Bermudo Ávila, 1983, p. 31).

Schmidt (1976, p. 24) señala que Marx emplea como sinónimos varios términos para definir a la realidad en su conjunto, sin excluir que el ser humano es parte de esa realidad, así: materia, naturaleza, sustancia natural, cosa natural, tierra, momentos existenciales objetivos del trabajo, condiciones objetivas o fácticas del trabajo. Para el marxismo, la materia o naturaleza es lo no subjetivo, «que no se disuelve en los modos de apropiación humana» (Schmidt, 1976, p. 18-24).

Desde la dialéctica se rechaza la existencia de una esencia última e inmutable de las cosas o de lo humano. La historia natural y la historia social van interrelacionadas, nuevamente considerando que la naturaleza es previa y el ser humano es parte de la misma, pero en condiciones muy particulares resultantes de su evolución como especie. La humanidad viene a ser «la parte de la naturaleza [que] adquiere conciencia de sí misma», no algo externo a ella ni desconectada con su futuro. Veamos la cita completa de Engels:

Y de los primeros animales se desarrollaron, esencialmente gracias a la diferenciación, incontables clases, órdenes, familias, géneros y especies, hasta llegar a la forma en la que el sistema nervioso alcanza su más pleno desarrollo, a los vertebrados, y finalmente, entre éstos, a un vertebrado, en que la naturaleza adquiere conciencia de sí misma, el hombre. (1961, p.15)

Este pensamiento, aunque frecuente en el avance científico, era y es contrario al pensamiento occidental hegemónico que, con una base judeo-cristiana, plantea al ser humano como la cúspide de la creación que debe dominar a la naturaleza como actor externo y que, además, no debe preocuparse por los límites de la naturaleza porque «ya Dios proveerá» (Mateo 6:26; Filipenses 4:19). El caso, entre tantos otros, de la pesca con zonas oceánicas en las cuales prácticamente han desaparecido los peces, es una de las actuales evidencias claras de lo contrario. Hoy es incuestionable que la naturaleza tiene límites en su disposición de bienes útiles para los seres humanos, que en distintos procesos, todos los recursos son no-renovables. ${ }^{1}$ En otras palabras, que la relación entre ecología y economía toma características nuevas e importantes. Se plantea de este modo, desde el marxismo, pero no solo desde allí, la idea de que la única 
forma de dominar a la naturaleza es conocer sus leyes y cumplirlas. En la Dialéctica de la Naturaleza de Engels (1961) lo dirá de manera muy clara y con ejemplos:

No debemos, sin embargo, lisonjearnos demasiado de nuestras victorias humanas sobre la naturaleza. Esta se venga de nosotros por cada una de las derrotas que le inferimos. Es cierto que todas ellas se traducen principalmente en los resultados previstos y calculados, pero acarrean, además, otros imprevistos, con los que no contábamos y que, no pocas veces, contrarrestan los primeros. [...] Y, de la misma o parecida manera, todo nos recuerda a cada paso que el hombre no domina, ni mucho menos, la naturaleza a la manera como un conquistador domina un pueblo extranjero, es decir, como alguien que es ajeno a la naturaleza, sino que formamos parte de ella con nuestra carne, nuestra sangre y nuestro cerebro, que nos hallamos en medio de ella y que todo nuestro dominio sobre la naturaleza y la ventaja que en esto llevamos a las demás criaturas consiste en la posibilidad de llegar a conocer sus leyes y de saber aplicarlas acertadamente. (p. 151-152)

Más allá de las metáforas de Engels, que de ninguna manera introducen en su obra el carácter de la naturaleza como un ser personalizado que toma decisiones, lo que en general se presenta es una interpretación desde la dialéctica que apunta a comprender que ningún sistema es completamente estático, lo que refleja algunos principios de la dialéctica de utilidad teórico y práctica: historicidad, interconexión universal, heterogeneidad, compenetración de los opuestos y niveles de integración (Lewontin y Levins, 2015).

\section{EL METABOLISMO SOCIEDAD-NATURALEZA}

Se afirma con certeza que Marx tomó el concepto de metabolismo a partir de los trabajos de investigadores de las ciencias naturales. En primer lugar, Martínez Alier y Schlüpmann (1991, pp. 86-89), señalan que el origen del término lo tomó Marx del químico agrícola Justus von Liebig, quién resalto la existencia de la circulación de nutrientes del suelo y el metabolismo animal (Von Liebig, 1840, citado por Martínez Alier y Schlüpmann, 1991), insistiendo en que reciclar las aguas residuales de las ciudades podría devolver al suelo agrícola los nutrientes perdidos.

Jacob Moleschot en su libro Fisiología del intercambio orgánico en las plantas y animales (1851) y otros estudios plantea desde una perspectiva materialista y empírica ese intercambio que responde al «ciclo eterno de la materia». En El ciclo de la vida (1864) (citado por Schmidt, 1976) Moleschot plantea que:

El movimiento de las materias primas, la vinculación y separación, la asimilación y desamilación, constituyen la esencia de toda actividad sobre la tierra [...]. El milagro reside en la eternidad de la substancia a través del cambio de forma, en el intercambio orgánico como fundamento principal de la vida terrestre [...]. (s. p.)

En tercer lugar y motivo de debate, está el «affaire Sergei Podolinsky», médico ucraniano que en 1880 envió un artículo suyo a Karl Marx en el que intentaba aplicar las teorías físicas del momento, especialmente las leyes de la termodinámica, a las teorías del valor y la plusvalía desarrollada por Marx. Paul Burkett y John. B. Foster (2004) demostraron que, al contrario 
de otras opiniones, el tema no fue despreciado por Marx y que éste no solo analizó el intercambio de materia, sino el de energía, tema central en la economía ecológica de nuestros días.

El trabajo es la relación entre la sociedad y la naturaleza. El metabolismo entre ambos fue siempre recíproco hasta el momento del capitalismo que genera una «fractura metabólica» (Foster, 2004) que se relaciona también como parte de la enajenación del trabajo. Comprender la fractura metabólica es un aporte importante del marxismo que forma parte de la crítica integral al sistema capitalista, importante para una estrategia de superación del capitalismo y particularmente del extractivismo (Sacher, 2015) y complementa la explicación de la alienación del trabajo.

La naturaleza es el cuerpo inorgánico del hombre; la naturaleza, en cuanto ella misma, no es cuerpo humano. Que el hombre vive de la naturaleza quiere decir que la naturaleza es su cuerpo, con el cual ha de mantenerse en proceso continuo para no morir. Que la vida física y espiritual del hombre está ligada con la naturaleza no tiene otro sentido que el de que la naturaleza está ligada consigo misma, pues el hombre es una parte de la naturaleza.

Como quiera que el trabajo enajenado (1) convierte a la naturaleza en algo ajeno al hombre, (2) lo hace ajeno de sí mismo, de su propia función activa, de su actividad vital, también hace del género algo ajeno al hombre; hace que para él la vida genérica se convierta en medio de la vida individual. En primer lugar hace extrañas entre sí la vida genérica y la vida individual, en segundo término convierte a la primera, en abstracta, en fin de la última, igualmente en su forma extrañada y abstracta. (Marx, 1974)

Esa alienación conlleva a que:

Con la preponderancia incesantemente creciente de la población urbana, acumulada en grandes centros por la producción capitalista, ésta por una parte acumula la fuerza motriz histórica de la sociedad y, por otra, perturba el metabolismo entre el ser humano y la tierra, esto es, el retorno al suelo de aquellos elementos constitutivos del mismo que han sido consumidos por el ser humano bajo la forma de alimentos y vestimenta, retorno que es condición natural eterna de la fertilidad permanente del suelo. Con ello destruye, al mismo tiempo, la salud física de los obreros urbanos y la vida intelectual de los trabajadores rurales. [...] Y todo progreso de la agricultura capitalista no es solo un progreso en el arte de esquilmar al obrero, sino a la vez en el arte de esquilmar el suelo; todo avance en el acrecentamiento de la fertilidad de este durante un lapso dado, un avance en el agotamiento de las fuentes duraderas de esa fertilidad. [...] La producción capitalista, por consiguiente, no desarrolla la técnica y la combinación del proceso social de producción sino socavando, al mismo tiempo, los dos manantiales de toda riqueza: la tierra y el trabajador. (Marx, 1973)

Volverá a esta idea en distintas partes. En el tomo III de El capital (Marx, 1973):

La gran propiedad de la tierra mina la fuerza de trabajo en la última región a que va a refugiarse su energía natural y donde se acumula como fondo de reserva para la renovación de la energía vital de las naciones: en la tierra misma. La gran industria y la gran agricultura explotada industrialmente actúan de un modo conjunto y crean una unidad. Si bien en un principio se separan por el hecho de que la primera devasta y arruina más bien la fuerza de trabajo y, por tanto, la fuerza natural del hombre y la segunda más directamente la fuerza natural de 
la tierra, más tarde tienden cada vez más a darse la mano, pues el sistema industrial acaba robando también las energías de los trabajadores del campo, a la par que la industria y el comercio suministran a la agricultura los medios para el agotamiento de la tierra. (p. 753)

Textos trascendentes, pero no los únicos, que contradicen la insistencia con la que se ha señalado que Marx consideraba que el trabajo era la única fuente de riqueza. Jorge Veraza (2011, p. 62) resalta cómo, de manera poco conocida, las primeras veintiocho páginas del primer manuscrito de 1844 , el texto se «distribuye» en tres columnas -El salario, La ganancia y La renta del suelo, de manera tal que continuamente se entrelazan. La importancia del análisis de la renta de la tierra no es solo remarcar que la naturaleza es una fuente de riqueza apropiada por el capital como una simple cosa, sino que «no se restringe ni teórica ni políticamente al economicismo ulterior del marxismo vulgar ("oficial") y fundamenta la alianza coordinada entre el proletariado y el campesinado $-\mathrm{y}$, con ésta, entre elementos capitalistas y precapitalistasen vista de la revolución comunista» (Veraza, 2011, p. 57).

Por otra parte, el texto de El capital copiado arriba, se relaciona con la explicación de cómo en el capitalismo las fuerzas productivas se transforman en fuerzas destructivas. Producción - creación - y destrucción entran en una dialéctica en la que paulatinamente lo segundo se va haciendo más evidente. Marx y Engels, en el Manifiesto del Partido Comunista, señalarán como:

Toda esta sociedad burguesa moderna, que ha hecho surgir como por encanto tan potentes medios de producción y de cambio, se asemeja al mago que ya no es capaz de dominar las potencias infernales que ha desencadenado con sus conjuros. (Marx y Engels, 1978, p. 116)

Y en el primer capítulo de la Ideología Alemana, Marx señalará que:

[...] En el desarrollo de las fuerzas productivas se llega a una fase en que surgen fuerzas productivas y medios de intercambio que, bajo las relaciones existentes, solo pueden ser fuente de males, que ya no son fuerzas productivas sino más bien destructivas - maquinaria y dinero-; y, a la vez, surge una clase condenada a soportar todos los inconvenientes de la sociedad sin gozar sus ventajas. (s. d.)

Las fuerzas productivas sociales, al decir de Veraza (2012), Marx las concibe como «formas orgánicas y de organización humana [...] que están constituidas por un factor objetivo y otro subjetivo, orgánicamente vinculados [...]». El aspecto subjetivo, que muchas veces se dice no fue atendido por Marx (1974, p. 654), en los manuscritos de 1844 se torna central en la fuerza productiva, tanto que señala que: «La pasión es la fuerza esencial del hombre que tiende enérgicamente hacia su objeto» (Veraza, 2012, p. 69).

La fractura metabólica se presenta bajo el capitalismo como una alteración destructiva en la relación entre sociedad y naturaleza, rompiendo los procesos de un sistema que está conectado por la circulación de elementos y flujo de energía. Es, en parte, el resultado de las capacidades transformadoras de los seres humanos que, a diferencia de los animales en los que prima la adaptación y los instintos, superan la interacción fisiológica con la naturaleza y la convierten en social. Por ello, ya no se trata de un metabolismo meramente natural, sino que requiere del factor consciente para su preservación o su destrucción. Por otra parte, la fractura 
metabólica históricamente está ligada a la producción de mercancías y el modo de producción capitalista que colocó a la naturaleza en condición de recursos económicos sometidos a la apropiación y acumulación.

\section{LO HISTÓRICO Y LO TRANSHISTÓRICO EN LA RELACIÓN SOCIEDAD NATURALEZA}

El ser humano es un ser productor. Está obligado a generar sus condiciones de supervivencia, a tomar lo necesario de la naturaleza, pero además tiene la capacidad de transformas sus herramientas, su organización, su hábitat. Esta realidad está presente durante toda la existencia de la especie. Tiene una condición permanente que llegaría a ser considerada transhistórica (Echeverría, 2011). Esto lo criticará Marx en Ricardo, quien universaliza la forma mercantil capitalista del trabajo bajo la forma de trabajo social per se, que termina en el fetichismo de la mercancía. Empleando textos de El capital, Pospone (2006) resalta:

Al contrario, entonces, que la interpretación común, Marx no asume la teoría del valor trabajo de Ricardo, ni la dota de más consistencia, ni la utiliza para probar que la ganancia es creada únicamente por el trabajo. Escribe una crítica de la economía política, una crítica inmanente de la propia teoría del valor trabajo clásica. (p. 54)

Pero considerando que somos parte de la naturaleza, para el marxismo la transformación de la misma realizada por el ser humano es al mismo tiempo la transformación del productor. Esa reciproca transformación nos permite comprender de qué manera el Hombre humaniza a la naturaleza - véanse los ejemplos de cómo importantes ecosistemas entre los cuales se encuentra la Amazonía existen gracias a la suma de procesos naturales con la intervención de poblaciones humanas, según lo demuestra Levis et al. (2017) —, así como las condiciones históricas del trabajo va también transformando al Hombre. Allí se encuentra el origen de la total conexión entre la historia natural por la historia social.

Pero esa condición transhistórica en tanto tal, cambia y tiene un carácter histórico que se expresa en la manera en la cual la sociedad se organiza, en el modo de producción. Cada una de ellos tiene una forma de acercarse a la naturaleza, de entenderla, de aprovecharla. Ello alterará incluso nuestra percepción de las necesidades, que se presentan diferenciadas en cada modo de producción, pasando de las básicas a las culturalmente pertenecientes y las forjadas comercialmente.

La relación de la humanidad con la naturaleza y el proceso de trabajo que la materializa, poseen un carácter eminentemente social, debido a que corresponden a relaciones sociales definidas y una división del trabajo ya dada. Por otra parte, el trabajo contribuye a la reproducción social y su reproducción y cambio son, en sí mismos, procesos sociales (Burkett 1999, p. 50).

Hay, por tanto, que resaltar que cuando se habla de la actual crisis civilizatoria, es decir, de la crisis de la civilización capitalista en sentido de la integralidad de sus componentes y factores de organización social (Isch, 2013), hay que tener presente que el sistema no se desarrolla al mismo ritmo en todos los lugares y que es importante considerar las diferencias de esa crisis de acuerdo a la formación económico social específica de un país -incluyendo los procesos productivos diferenciados del capitalismo- y las características étnico culturales presentes. 
De manera que la crisis de relacionamiento de la sociedad con la naturaleza o la «fractura metabólica» no tendrá idénticas expresiones en todos los países.

Un rasgo común a las distintas formas de relacionamiento, social y socionatural en el capitalismo, lo podríamos encontrar en una expresión de David Harvey (2007, p. 137-140): el capitalismo es la fábrica de la fragmentación de la realidad. En ella siempre se busca el crecimiento sin considerar las consecuencias y siempre se lo relaciona con la idea de «progreso» como un hecho inevitable.

\section{LA TEORÍA DEL VALOR}

La fractura metabólica en el capitalismo tiene que ser entendida además desde la teoría del valor y la explicación integral de la mercancía. Lo visto anteriormente demuestra que la naturaleza, para Marx, es una fuente de valores de uso y puede ser ella misma un valor de uso. Cada bien natural puede ser valorado desde distintas perspectivas, entre las cuales la económica como valor de cambio es tan solo una entre tantas como la cultural, espiritual religiosa, estética, recreacional, etc.

Esto, una vez más, nos lleva a comprender que existe una impronta de cada cultura en la relación con la naturaleza que nos dirá qué vamos a consumir, cómo lo haremos, los simbolismos y rituales que acompañarán al consumo, la racionalidad implicada. El capitalismo impondrá una concepción que se orienta hacia la acumulación de capitales en pocas manos y que, para ello, impulsará el consumismo, es decir el consumo de lo innecesario en cantidades innecesarias, generando una sobre explotación de la naturaleza - junto a la sobre explotación que destroza la salud de los trabajadores-y una sobre «producción» de desechos que la naturaleza no podrá procesar.

El objetivo de la acumulación diferencia al capitalismo de otras organizaciones sociales de producción. Tomemos por ejemplo la extracción de mineras en los pueblos andinos antes de la conquista: obtenían cantidades relativamente pequeñas de oro como un valor de uso que servía principalmente para ritos religiosos, llegando al caso de volverlos a enterrar o arrojar a lagos sagrados como ofrenda luego de haberlos transformado en obras de arte. Allí no hay acumulación. Esto no es de ninguna manera equiparable con la acumulación originaria del capital ni con el extractivismo actual, que se caracteriza por extraer grandes cantidades de mineral, orientado a la exportación, como un valor de cambio con el cual se alimenta el modelo de acumulación de la riqueza en pocas manos. Y el afán de lucro acelerará este círculo vicioso de lograr más mineral, para acumular más, con graves e innegables consecuencias sociales y ambientales.

Marx cuestiona esa lógica:

La naturaleza se transforma en puro objeto para el hombre, en pura cosa utilitaria; deja de ser reconocida en tanto potencia para sí. Y el mismo conocimiento teórico de sus leyes autónomas aparece solamente como argucia para someterla a sus propias necesidades, sea como objeto de consumo o como vehículo de producción. (s. d.)

Adicionalmente se puede discutir el uso de categorías como «recursos naturales» referida desde 
la economía a los elementos de la naturaleza que pueden brindar ganancias; así como de «servicios ecosistémicos» que, desde el mismo origen define a los beneficios visibles que nos brindan esos elementos o los ecosistemas. Son categorías que responden a esa visión utilitaria de la naturaleza y que responden en nuestros días a un «ambientalismo de mercado», que no es sino la propuesta neoliberal de solucionar los conflictos ambientales de usufructo de la naturaleza a través del mercado (Isch y Gentes, 2006). Más aún cuando los llamados «servicios ambientales» son un resultado natural, sin trabajo humano, por lo que se constituyen mercados a partir de pseudomercancías, tal y como sucede en el mercado de carbono y su intento de comercializar moléculas de este elemento de la tabla periódica (Lohmann, 2012). Convertir los bienes naturales en recursos y las funciones de especies y ecosistemas en «servicios», es cuestionado además por el antropocentrismo presente y que involucra, una vez más, una relación entre sociedad y naturaleza en término de opuestos o de enemigos (Acosta y Martínez, 2011).

El fetichismo de la mercancía, analizado por Marx, es la manera en la que se transfieren cualidades humanas o se entregan cualidades mágicas a las mercancías. Se la aprovecha para señalar lo supuestamente inevitable de mantener una manera de producción y de consumo. Culturalmente, se impulsa el afán de lucro, el poseer o consumir mercancías nos hace humanos superiores frente a los que no lo hacen. El ser humano se cosifica al mismo tiempo que la mercancía y el mercado se tornan en el centro de la vida social. Estas visiones están en el centro del extractivismo y, con ellas, se justifica su ejercicio de la violencia legalizada.

Un elemento de crítica es destacar el valor de no-uso que pueden tener las mercancías que es parte de la teoría del valor elaborada por Marx y se constituye de la existencia del bien igual que un legado que se hereda - y de la posibilidad de optar que hacer con ese bien existente. «Todas las mercancías», enseña Marx, «son para su poseedor no-valores de uso y valores de uso para los no poseedores». En consecuencia, antes de poder realizarse como valores de uso, las mercancías tienen primeramente que realizarse como valores (Kruse, s. f.).

El valor de no uso se define como un valor de existencia no vinculado con ningún uso y ninguna búsqueda de utilidad, ni de forma consuntiva o no consuntiva del bien ambiental. El valor de no-uso tiene especial importancia cuando se presentan propuestas como la Iniciativa Yasuní-ITT (2007-2013) que proponía dejar el crudo bajo suelo y priorizar la protección ambiental y de los pueblos en aislamiento voluntario. Su análisis se desarrolla desde la economía ecológica basada en el cálculo de costo-oportunidad y la contabilidad de externalidades, que nada tiene que ver con la economía ambiental que en un sistema abierto e interdisciplinario, a partir de los intercambios de materia y flujos de energía, estudia las relaciones entre distintos sistemas -ecológicos, económicos, sociales y culturales-.

La consideración de las distintas valoraciones de la naturaleza desde perspectivas ecológicas, culturales, paisajísticas, económicas, espirituales y más, abre las posibilidades de crítica de la economía ortodoxa, y una de sus concreciones tiene puntos de encuentro con la economía ecológica. Sin embargo, allí donde se ha impulsado el mercado de «servicios ecosistémicos», ha sido empleada también para establecer mecanismos de valoración económica que permitan el ambientalismo de mercado apoyado en la economía ambiental, la que coincide con la ortodoxia en considerar a los daños ambientales fundamentalmente como una «externalidad» al mercado. 
En este punto, es importante destacar que la teoría marxista de la renta de la tierra, necesaria para comprender la comercialización de ciertas pseudomercancías de origen natural, es también una conclusión de su teoría del valor. La renta de la tierra es una aplicación de la teoría del valor a «una parte de la naturaleza que puede ser monopolizable» (Folladori, 1996, p. 5) a la vez que permite explicar la degradación del suelo en la búsqueda de mayores rendimientos económicos.

\section{A MODO DE CONCLUSIÓN: CRISIS AMBIENTAL Y LUCHA DE CLASES}

Como se sabe, Marx y Engels señalaron en sus propias palabras que la historia de todas las sociedades que han existido hasta nuestros días «es la historia de las luchas de clases» (Marx y Engels, 1978). Esto no fueron los primeros en plantearlo, sino que el aporte marxista estaría en demostrar que «la lucha de clases lleva necesariamente a la dictadura del proletariado» (Marx y Engels, 1979).

En términos ambientales, la lucha de clases se presenta en los procesos de apropiación, transformación y comercialización de la naturaleza convertida en valor. El escenario actual es un proceso de «colonización por el capital de los procesos naturales de reproducción de la vida — vegetal, animal y humana-», lograda con una «nueva composición tecnológica de los procesos de producción que hace más densa y sofisticada a la dominación del trabajo muerto sobre el trabajo vivo» y que, a su vez, permite «un nuevo ciclo de despojo de bienes comunes» (Roux, 2012).

El despojo continua la historia de la acumulación originaria en los territorios en los cuales el capitalismo no podía expresarse de manera plena por la existencia de formas civilizatorias indígenas o culturales de alta valoración de lo común. Con las particularidades de nuestros días, David Harvey señalaría que se trata de una «acumulación por desposesión» que, no hay que olvidar, enajena a poblaciones enteras de su hábitat y forma de vida, dejándoles en condiciones de poseedores únicamente de fuerza de trabajo para venderla en el mercado. Afectados serán, en primer lugar, las poblaciones indígenas y campesinas que mantuvieron sus territorios y recursos al margen de la plena explotación capitalista; y lo serán las mujeres, que, se ha visto en todo lugar, reciben mayores impactos y se convierten en las mejores defensoras de la naturaleza y la vida.

La lucha de clases, entonces, está cruzada por relaciones étnicas y también por relaciones de género y otras que no pueden ser descuidadas. El despojo incluye bienes comunes no materiales como la cultura y los valores colectivos, se justifica por el menosprecio de racista y etnocentrista al otro y por la naturalización del discrimen a las mujeres.

Allí hay una confrontación de clases y diversos grupos humanos que puede adquirir distintas expresiones y formas, pero que lleva a la resistencia, por un lado, y al uso de los aparatos represivos y de control social, por el otro. El Estado en manos de la burguesía cumple su función de garante de los intereses de su clase y hace de la violencia oficial una condición inseparable del despojo y el extractivismo. El Estado, aliado a las empresas nacionales o transnacionales, coloca sus intereses por encima de los derechos de los pobladores despojados de sus territorios, formas de vida, cultura. 
En última instancia, la situación actual será de una encrucijada expresada por Engels y resaltada por Rosa Luxemburgo: o socialismo o barbarie. La manera necesaria de resolver los problemas ambientales está en el socialismo como transición hacia la sociedad sin clases, dado que el capitalismo, incluso con propuestas de economía verde, no puede escapar de su lógica de explotación del trabajador y expoliación de la tierra a niveles que se han convertido insostenibles a mediano plazo.

La imposibilidad de compatibilizar el capitalismo con una real protección ambiental ha sido denunciada no solo por marxistas. La observación de la lucha entre productores locales y grandes transnacionales, entre poblaciones amigas del ambiente y empresas que impulsan su acelerada destrucción, entre los que acumulan cada vez más capital y los despojados que se quedan sin nada, se puede realizar todos los días.

La importancia del marxismo es que nos da una alternativa posible a nivel local y global a los problemas sociales y dentro de ellos, a la necesaria restitución del metabolismo entre la sociedad y naturaleza. Y éste es un propósito revolucionario que, junto a otros, debe orientar la economía de un socialismo verdadero, científico, en las condiciones de nuestro tiempo.

\section{NOTAS}

1 La concepción de «recurso natural», en contraposición al de bienes naturales, está dada por una posición mercantilista. Hoy se asume que incluso el agua dulce disponible para los seres humanos, considerada como renovable gracias al ciclo natural del agua, ya no la es: hemos afectado ese ciclo provocando el cambio climático; la contaminación reduce de manera continua el agua utilizable; mientras las formas diversas de mercados del agua y de acaparamiento del líquido vital hacen que sea escasa para amplios sectores poblacionales, incluso en países con una buena dotación del líquido, como Ecuador.

2 Por supuesto, el problema de la fertilidad del suelo ha sido estudiado a lo largo de los años señalando el carácter multifactorial del mismo. Un análisis de este punto, en relación al concepto de «fractura metabólica», se encuentra en el trabajo de William Sacher (2015).

\section{REFERENCIAS}

Acosta, A. y Martínez, E. (2011). La naturaleza con derechos. De la filosofía a la política. Quito, Ecuador: Fundación Rosa Luxemburg y Abya Yala.

Alimonda, H. (2012). Ecología política. Naturaleza, sociedad y utopía. Buenos Aires, Argentina: CLAcso. Bebbington, A. (2007). Elementos para una ecología política de los movimientos sociales y el desarrollo territorial en zonas mineras. En A. Bebbington (Ed.), Minería, movimientos sociales y respuestas campesinas (23-46). Lima, Perú: IEP-CEPES.

Bermudo Ávila, J. M. (1981). Engels contra Marx: (el antiengelsianismo en el marxismo eurooccidental). Barcelona, España: Publicaciones de la Universidad de Barcelona.

Broecker, W. S. (1975). Climatic change: Are we on the brink of a pronounced global warming? Science, 189(4201), 460-463.

Burkett, P. (1999). Marx and nature. A red and green perspective. New York, UsA: Co. Publishing Palgrave-Macmillan.

Burkett, P. y Foster, J. B. (2004). Ecological economics and classical marxism: The «Podolinsky Business» Reconsidered. Organization and Environment, 17(1), 32-6o. 
Castellanos Suárez, J. A. (2015). El carácter no ontológico de la naturaleza en Marx. El sentido de Alfred Schmidt e implicaciones en la Escuela de Frankfurt. Asuntos Económicos y Administrativos, 29, 31-42. Echeverría, B. (2011). Crítica de la modernidad capitalista. Antología. La Paz, Bolivia: Vicepresidencia del Estado Plurinacional de Bolivia.

Engels, F. (1974 [1886]). Carta de F. Engels a Adolph Sorge del 29 de noviembre de 1886. En C. Marx y F. Engels, Obras escogidas: Moscú, URss: Editorial Progreso.

Engels, F. (1974 [1895]). Carta a Werner Sombart del 11 de marzo de 1895. En C. Marx y F. Engels, Obras escogidas: Moscú, URss: Editorial Progreso.

Engels, F. (1961). La dialéctica de la naturaleza. México DF, México: Editorial Grijalbo.

Foster, J. B. (2004). La ecología de Marx. Materialismo y naturaleza. Barcelona, España: El Viejo Topo.

Gudynas, E. (2013). Correa, minería y Marx: hoy, en América Latina, Marx ¿sería extractivista? Celidin Libre. Recuperado de: https://celendinlibre.wordpress.com/tag/joan-martinez-alier/

Grupo Intergubernamental de Expertos sobre el Cambio Climático (IPCC) (2007). Informe del Grupo de Trabajo I del Grupo Intergubernamental de Expertos sobre el Cambio Climático de Naciones Unidas. Recuperado de http://www.ipcc.ch/home_languages_main_spanish.shtml

Isch, E. (2011). Módulo de capacitación sobre cambio climático. Quito, Ecuador: The Nature Conservancy, COICA, CAMAREN.

Isch, E. (2013, octubre). Crisis civilizatoria y sus expresiones en Latinoamérica. Ponencia presentada en la I Asamblea del Movimiento por la salud de los pueblos Latinoamérica. Cuenca, Ecuador.

Isch, E. (2017). Tejiendo resistencias por la vida. Módulos pedagógicos en defensa de la Pachamama. Recuperado de http://www.estudiosecologistas.org/documentos/publicaciones/MODULO\%2O ECOLOGIA\%2OPOLITICA\%2OPARA\%2OWEB_2.pdf

Isch, E. y Gentes, I. (Eds.) (2006). Agua y servicios ambientales. Visiones críticas desde los Andes. Quito, Ecuador: WALIR, Camare, Abya Yala.

Kolakowski, L. (1983). Las principales corrientes del marxismo. Madrid, España: Alianza Editorial.

Kruse, H. A. (s. f.). Marx y los poseedores de mercancías. Redacción Popular. Recuperado de http://www. redaccionpopular.com/articulo/marx-y-los-poseedores-de-mercancias?page=1

Levis, C., Costa, F. R., Bongers, F., Peña-Claros, M., Clement, C. R., Junqueira, A. B. y Castilho, C. V. (2017). Persistent effects of pre-Columbian plant domestication on Amazonian forest composition. Science, 355, 925-931.

Lewontin, R. y Levins, R. (2015). El biólogo dialéctico. Buenos Aires, Argentina: RyR.

Lohmann, L. (2012). Mercados de carbono. La neoliberalización del clima. Quito, Ecuador: Abya Yala.

Martínez Alier, J. (2013). Marx y el extractivismo del presidente Correa. Celendin libre. Recuperado de https://celendinlibre.wordpress.com/tag/joan-martinez-alier/

Martínez Alier, J. (2005). El ecologismo de los pobres: conflictos ecológicos y lenguajes de valoración. Barcelona, España: Icaria.

Martínez Alier, J. y K. Schlüpmann. (1991). La ecología y la economía. México DF, México: Fondo de Cultura Económica.

Marx, K. (1973). El capital. México DF, México: Fondo de Cultura Económica.

Marx, K. (1974). Manuscritos económicos y filosóficos de 1844. Madrid, España: Alianza Editorial.

Marx, K. y Engels, F. (1978). El manifiesto del Partido Comunista (Obras escogidas, tomo I). México DF, México: Editorial Progreso. 
Milián, R. (2007). Historia de la ecología. Ciudad de Guatemala, Guatemala: Universidad de San Carlos de Guatemala.

Piedra Arencibia, R. (2015, septiembre). La teodicea del marxismo. El antiengelsianismo y su función histórica. Temas, 83, 96-102.

Pospone, M. (2006). Tiempo trabajo y dominación social. Una reinterpretación de la teoría crítica de Marx. Madrid, España: Editorial Marcial Pons.

Sacher, W. (2015). La «fractura metabólica» de John Bellamy Foster: ¿Qué aportes para una teoría ecomarxista? Actuel Marx / Intervenciones, 19, 33-60.

Schmidt, A. (1976). El concepto de naturaleza en Marx. México DF, México: Siglo xxi.

Roux, R. (2012). México: despojo universal, desintegración de la república y nuevas rebeldías. Theomai, 26, (s. d.).

Veraza, J. (2012). Karl Marx y la técnica desde la perspectiva de la vida. Por una teoría marxista de las fuerzas productivas. México DF, México: Ítaca. 